\title{
SOME ECOLOGICAL NOTES ON THE BUTTERFLIES OF VISAKHAPATNAM, ANDHRA PRADESH
}

\section{A.J. Solomon Raju ${ }^{1}$, S. Purnachandra Rao and V.Ezradanam}

Department of Environmental Sciences, Andhra University, Visakhapatnam, Andhra Pradesh 530003, India.

IEmail:ajsraju@yahoo.com

Visakhapatnam is situated on the east coast $\left(17^{\circ} 42^{\prime} \mathrm{N} ; 82^{\circ} 18^{\prime} \mathrm{E}\right)$ of India. It consists of hills/ghats, the plains and the sea coast. The area receives rain during south-west monsoon and also retreating showers. The average temperature ranges from $18^{\circ} \mathrm{C}$ in January to $40^{\circ} \mathrm{C}$ in May. The area shows a graded diversity in species composition and in the quality of vegetation. The plain areas show mostly scrub jungles and the herbaceous vegetation thrives well during rainy season. Visakhapatnam is a rapidly expanding industrial city which has taken a heavy toll on the quality and quantity of wild vegetation. The various anthropogenic activities are constantly posing a great threat to the still available wild flora and fauna. The surviving plant and animal species have only two options - either to adapt to degrading and disturbing habitats or to disappear permanently.

The present study is an attempt to provide a checklist of butterflies based on a two-year field study from October 2000 to October 2002 in Visakhapatnam (Table 1). The status of each butterfly species is also given in Table 1. Earlier, there was a study on the butterflies of Visakhapatnam by Reddi and Bai (1985). These authors reported that there are only 46 butterfly species in Visakhapatnam. Further, they wrongly reported the status of some butterflies. Later, they added four more species to the list, while making a rare butterfly species, Spindasis vulcanus (Reddi \& Bai, 1985) as a common one (Ramana et al., 2001). These reports show many errors. In view of these confusing records with incomplete checklist of butterflies for Visakhapatnam, the present study was made to provide a comprehensive list of butterflies together with their status. We make a special mention that there are atleast 10 more butterfly species which may have escaped our eyes. We are still continuing our studies on the butterflies of Visakhapatnam with reference to their host-plants for egglaying and larval feeding and period of occurrence. As and when we finish our studies, we will report our findings.
Our studies during the period indicate that the habitats where butterflies were found and captured are disturbed areas and are strongly influenced by man's activities. These range from city lots to pastures, abandoned fields, road sides, parks, urban plantings, etc. Many of these habitats have been found to support butterflies because of the occurrence of weedy exotics like Lantana camara, Hyptis suaveolens, Antigonon leptopus, etc. and a wide variety of herbaceous species. These plant species with their colourful flowers and also colour shifts as in Lantana flowers attract butterflies even from a long distance and provide them with floral nectar. More than 30 butterfly species were found utilising the floral nectar of Lantana. Although Lantana camara is an alien invasive species and a proven competitor for native grasses, herbs and shrubs, its showy, colourful flowers serve as alternate floral nectar source for butterflies. Although its abundant growth in Visakhapatnam and also in the entire tropical belt seems like a provisioning post and a nectar source for adult butterflies (Raju \& Reddi, 1995), the negative effects of this alien invasive on native food and host plants of butterflies could affect the long-term sustainibility of butterfly populations in the wild.

The study reveals that there are 68 butterfly species occurring in Visakhapatnam, representing all five families of the suborder Rhopalocera of the order Lepidoptera. The Papilionidae is represented by three genera with 10 species, Pieridae by nine genera with 13 species, Nymphalidae by 14 genera with 23 species, Lycaenidae by 17 genera, each with one species only and Hesperiidae by five genera, each with one species only. In Papilionidae, the genus Pachliopta is represented by two species, Graphium by three species, Papilio by five species. In Pieridae, Catopsilia and Eurema, each represented by two species, Colotis by three species and all other genera by one species only. The Nymphalidae genera, Mycalesis, Euthalia, Hypolimnas, Tirumala and Danaus are represented by two species each, Junonia by five species and all others by one species only. In all five families, Papilio and Junonia genera are best represented than any other genera in Visakhapatnam.

Our study shows that the sustained interference and disturbance seem to affect the occurrence and numerical strength of each butterfly species. If this situation goes unabated, the abundant butterflies may become rare and the less abundant ones could disappear permanently. Further, the decline in the number of butterflies largely allows inbreeding which becomes fatal in course of time. Modified habitats with reduced plant cover contribute to warm conditions and these conditions might allow some butterflies to extend their distribution to different habitats. The butterflies which control certain plant pests, if decline in number or disappear from the habitat, plants too get affected because of the unchecked plant pests. Therefore, the very presence of butterflies in species and numbers may be taken as an indication of the health of the habitat(s) (Raju \& Rao, 2002).

Manuscript received 9 November 2002; $\quad$ Revised received 27 January 2003; $\quad$ Finally accepted 20 April 2003 
Table 1. Butterflies of Visakhapatnam, Andhra Pradesh

\begin{tabular}{|c|c|c|c|c|}
\hline Scientific name & English name & Status & Collection numbers & No. of specimens \\
\hline \multicolumn{5}{|l|}{ Papilionidae } \\
\hline Graphium agamemnon Linnaeus & Tailed Jay & VC & VSKPPAP1:1 - VSKPPAP1:38 & 38 \\
\hline Graphium doson C \& R Felder & Common Jay & $\mathrm{R}$ & VSKPPAP2:1 - VSKPPAP2:3 & 3 \\
\hline Graphium nomius Esper & Spot Swordtail & $\mathrm{R}$ & VSKPPAP3:1 - VSKPPAP3:4 & 4 \\
\hline Pachliopta aristolochiae Fabricius & Common Rose & $\mathrm{C}$ & VSKPPAP4:1 - VSKPPAP4:28 & 28 \\
\hline Pachliopta hector Linnaeus & Crimson Rose & $\mathrm{C}$ & VSKPPAP5:1 - VSKPPAP5:32 & 32 \\
\hline Papilio demoleus Linnaeus & Lime Butterfly & $\mathrm{C}$ & VSKPPAP6:1 - VSKPPAP6:29 & 29 \\
\hline Papilio polymnestor Cramer & Blue Mormon & $\mathrm{R}$ & VSKPPAP7:1 - VSKPPAP7:5 & 5 \\
\hline \multicolumn{5}{|l|}{ Papilio polytes Linnaeus } \\
\hline Papilio polytes polytes Linnaeus & Common Mormon & C & VSKPPAP8A:1 - VSKPPAP8A:32 & 32 \\
\hline Papilio polytes romulus Cramer & Common Mormon & C & VSKPPAP8B:1 - VSKPPAP8B:26 & 26 \\
\hline Papilio polytes stichius Hubner & Common Mormon & UC & VSKPPAP8C:1 - VSKPPAP8C:10 & 10 \\
\hline \multicolumn{5}{|l|}{ Pieridae } \\
\hline Anaphaeis aurota Fabricius & Pioneer or Caper white & $\mathrm{C}$ & VSKPPIE1:1 - VSKPPIE1:29 & 29 \\
\hline Appias albina Boisduval & Common Albatross & UC & VSKPPIE2:1 - VSKPPIE2:13 & 13 \\
\hline Catopsilia pomona Fabricius & Common Emigrant & VC & VSKPPIE3:1 - VSKPPIE3:40 & 40 \\
\hline Catopsilia pyranthe Linnaeus & Mottled Emigrant & VC & VSKPPIE4:1 - VSKPPIE4:45 & 45 \\
\hline Cepora nerissa Fabricius & Common Gull & UC & VSKPPIE5:1 - VSKPPIE5:12 & 12 \\
\hline Colotis danae Fabricius & Crimson Tip & C & VSKPPIE6:1 - VSKPPIE6:34 & 34 \\
\hline Colotis eucharis Fabricius & Plain Orange Tip & $\mathrm{C}$ & VSKPPIE7:1 - VSKPPIE7:31 & 31 \\
\hline Colotis fausta Olivier & Large Salmon Arab & $\mathrm{R}$ & VSKPPIE8:1 - VSKPPIE8:3 & 3 \\
\hline Delias eucharis Drury & Common Jezebel & VR & VSKPPIE9:1 - VSKPPIE9:2 & 2 \\
\hline Eurema blanda Boisduval & Three Spot Grass Yellow & $\mathrm{C}$ & VSKPPIE10:1 - VSKPPIE10:29 & 29 \\
\hline Eurema hecabe Linnaeus & Common Grass Yellow & VC & VSKPPIE11:1 - VSKPPIE11:38 & 38 \\
\hline Leptosia nina Fabricius & Psyche & VC & VSKPPIE12:1 - VSKPPIE12:39 & 39 \\
\hline Pareronia valeria Cramer & Common Wanderer & C & VSKPPIE13:1 - VSKPPIE13:32 & 32 \\
\hline \multicolumn{5}{|l|}{ Lycaenidae } \\
\hline$\overline{\text { Arhopala amantes Hewitson }}$ & Large Oak Blue & VR & VSKPLYC1:1 & 1 \\
\hline Azanus ubaldus Cramer & Bright Babul Blue & $\mathrm{C}$ & VSKPLYC2:1 - VSKPLYC2:30 & 30 \\
\hline Castalius rosimon Fabricius & Common Pierrot & $\mathrm{C}$ & VSKPLYC3:1 - VSKPLYC3:28 & 28 \\
\hline Catochrysops strabo Fabricius & Forget-me-not & UC & VSKPLYC4:1 - VSKPLYC4:12 & 12 \\
\hline Chilades pandava Horsfield & Plains Cupid & UC & VSKPLYC5:1 - VSKPLYC5:11 & 11 \\
\hline Curetis thetis Drury & Indian Sunbeam & $\mathrm{R}$ & VSKPLYC6:1 - VSKPLYC6:4 & 4 \\
\hline Euchrysops cnejus Fabricius & Gram Blue & C & VSKPLYC7:1 - VSKPLYC7:24 & 24 \\
\hline Everes lacturnus Godart & Indian Cupid & $\mathrm{C}$ & VSKPLYC8:1 - VSKPLYC8:29 & 29 \\
\hline Jamides celeno Cramer & Common Cerulean & C & VSKPLYC9:1 - VSKPLYC9:26 & 26 \\
\hline Prosotas nora C \& R Felder & Common Lineblue & C & VSKPLYC10:1 - VSKPLYC10:31 & 31 \\
\hline Pseudozizeeria maha Kollar & Pale Grass Blue & $\mathrm{C}$ & VSKPLYC11:1 - VSKPLYC11:32 & 32 \\
\hline Rapala iarbus Fabricius & Indian Red Flash & $\mathrm{R}$ & VSKPLYC12:1 - VSKPLYC12:2 & 2 \\
\hline Rathinda amor Fabricius & Monkey Puzzle & UC & VSKPLYC13:1 - VSKPLYC13:11 & 11 \\
\hline Spalgis epius Westwood & Apefly & UC & VSKPLYC14:1 - VSKPLYC14:9 & 9 \\
\hline Spindasis vulcanus Fabricius & Common Silverline & C & VSKPLYC15:1 - VSKPLYC15:26 & 26 \\
\hline Talicada nyseus Guerin Meneville & Red Pierrot & $\mathrm{C}$ & VSKPLYC16:1 - VSKPLYC16:28 & 28 \\
\hline Zizeeria karsandra Moore & Dark Grass Blue & UC & VSKPLYC17:1 - VSKPLYC17:13 & 13 \\
\hline \multicolumn{5}{|l|}{ Nymphalidae } \\
\hline$\overline{\text { Acraea violae Fabricius }}$ & Tawny Coster & VC & VSKPNYM1:1 - VSKPNYM1:37 & 37 \\
\hline Ariadne merione Cramer & Common Castor & VC & VSKPNYM2:1 - VSKPNYM2:36 & 36 \\
\hline Danaus chrysippus Linnaeus & Plain Tiger & VC & VSKPNYM3:1 - VSKPNYM3:45 & 45 \\
\hline Danaus genutia Cramer & Striped or Common Tiger & UC & VSKPNYM4:1 - VSKPNYM4:13 & 13 \\
\hline Elymnias hypermenstra Linnaeus & Common Palmfly & $\mathrm{R}$ & VSKPNYM5:1 - VSKPNYM5:2 & 2 \\
\hline Euploea core Cramer & Common Indian Crow & VC & VSKPNYM6:1 - VSKPNYM6:40 & 40 \\
\hline Euthalia aconthea Cramer & Common Baron & UC & VSKPNYM7:1 - VSKPNYM7:10 & 10 \\
\hline
\end{tabular}




\begin{tabular}{|c|c|c|c|c|}
\hline Scientific name & English name & Status & Collection numbers & No. of specimens \\
\hline Euthalia nais Forster & Baronet & C & VSKPNYM8:1 - VSKPNYM8:32 & 32 \\
\hline Hypolimnas bolina Linnaeus & Great Eggfly & UC & VSKPNYM9:1 - VSKPNYM9:11 & 11 \\
\hline Hypolimnas misippus Linnaeus & Danaid Eggfly & VC & VSKPNYM10:1 - VSKPNYM10:38 & 38 \\
\hline Junonia almana Linnaeus & Peacock Pansy & UC & VSKPNYM11:1 - VSKPNYM11:10 & 10 \\
\hline Junonia hierta Fabricius & Yellow Pansy & C & VSKPNYM12:1 - VSKPNYM12:31 & 31 \\
\hline Junonia iphita Cramer & Chocolate Pansy & UC & VSKPNYM13:1 - VSKPNYM13:10 & 10 \\
\hline Junonia lemonias Linnaeus & Lemon Pansy & VC & VSKPNYM14:1 - VSKPNYM14:34 & 34 \\
\hline Junonia orithya Linnaeus & Blue Pansy & UC & VSKPNYM15:1 - VSKPNYM15:13 & 13 \\
\hline Melanitis leda Linnaeus & Common Evening Brown & UC & VSKPNYM16:1 - VSKPNYM16:9 & 9 \\
\hline Melanitis phedima Stoll & Dark Evening Brown & UC & VSKPNYM17:1 - VSKPNYM17:10 & 10 \\
\hline Mycalesis perseus Fabricius & Common Bush Brown & UC & VSKPNYM18:1 - VSKPNYM18:14 & 14 \\
\hline Mycalesis subdita Moore & Tamil Bush Brown & UC & VSKPNYM19:1 - VSKPNYM19:13 & 13 \\
\hline Neptis hylas Moore & Common Sailor & $\mathrm{C}$ & VSKPNYM20:1 - VSKPNYM20:28 & 28 \\
\hline Parantica aglea Stoll & Glassy Tiger & UC & VSKPNYM21:1 - VSKPNYM21:12 & 12 \\
\hline Phalanta phalantha Drury & Common Leopard & C & VSKPNYM22:1 - VSKPNYM22:31 & 31 \\
\hline Tirumala limniace Cramer & Blue Tiger & $\mathrm{C}$ & VSKPNYM23:1 - VSKPNYM23:26 & 26 \\
\hline Tirumala septentrionis Butler & Dark Blue Tiger & UC & VSKPNYM24:1 - VSKPNYM24:10 & 10 \\
\hline Ypthima asterope Klug & Common Three-Ring & UC & VSKPNYM25:1 - VSKPNYM25:10 & 10 \\
\hline \multicolumn{5}{|l|}{ Hesperiidae } \\
\hline 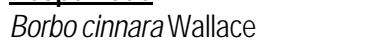 & Rice Swift & UC & VSKPHES1:1 - VSKPHES1:28 & 28 \\
\hline Halpe homolea Hewitson & Indian Ace & UC & VSKPHES2:1 - VSKPHES2:12 & 12 \\
\hline Pelopidas mathias Fabricius & Small Branded Swift & C & VSKPHES3:1 - VSKPHES3:33 & 33 \\
\hline Saustus gremius Fabricius & Indian Palm Bob & $\mathrm{C}$ & VSKPHES4:1 - VSKPHES4:29 & 29 \\
\hline Spialia galba Fabricius & Indian Skipper & $\mathrm{C}$ & VSKPHES5:1 - VSKPHES5:26 & 26 \\
\hline
\end{tabular}

Butterflies collected during the course of this study are maintained in the Department of Environmental Sciences, Andhra University, Visakhapatnam.

\section{Acknowledgement}

We thank Dr. N. Chaturvedi, Curator, Bombay Natural History Society, Bombay for identifying some butterfly specimens. We also extend thanks to Mr. Md. Hanifuddin and Mr. B. Madhu for collecting butterflies with great care and patience.

\section{References}

Raju, A.J.S. and C.S. Reddi (1995). Flower colour shifts and pollination in Lantana camara L. (Verbenaceae). Journal of Palynology 31: 275-289.

Raju, A.J.S. and S.P. Rao (2002). A case study on the decline of butterfly colonies in degraded habitats of Visakhapatnam. Bulletin of Andhra University Research Forum 7(10): 57-59.

Ramana, S.P.V., J.B. Atluri and C.S. Reddi (2001). New additions to the butterflies of Visakhapatnam. Insect Environment 6(4): 187.

Reddi, C.S. and G.M. Bai (1985). Butterflies of Visakhapatnam, their seasonality and relative abundance. Kingfisher Special Issue, pp. 1-2. Andhra Pradesh Natural History Society, Visakhapatnam. 